\title{
A NOTE ON FOURIER-STIELTJES TRANSFORMS ${ }^{1}$
}

\section{W. F. EBERLEIN}

Given a locally compact Abelian group $G$, let $\mu$ be a bounded Radon measure on the character group $G^{*}$. Then $\sum_{\nu \in G^{*}}|\mu\{y\}|<\infty$ -i.e. at most a countable set of points $\left(y_{n}\right)$ are of nonzero $\mu$-measure and $\sum_{n}\left|\mu\left\{y_{n}\right\}\right|<\infty . \mu$ thus admits the decomposition

$$
\mu=\sum_{n} \mu\left\{y_{n}\right\} \mu_{n}+\nu,
$$

where $\mu_{n}$ is a unit measure concentrated at the point $y_{n}$, and $\nu$ is a measure vanishing on single points (continuous measure). The Fourier transform of $\mu$,

$$
\widetilde{\mu}(x)=\int_{G^{*}}(x,-y) d \mu(y)=\sum_{n} \mu\left\{y_{n}\right\}\left(x,-y_{n}\right)+\int_{G^{*}}(x,-y) d \nu(y),
$$

is a bounded uniformly continuous function defined on $G$-in fact, a weakly almost periodic (w.a.p.) function. (Cf. $[1 ; 2]) .{ }^{2}$ We show that some theorems of Wiener [7], Beurling and Helson [3], and Hewitt [4] are contained in the following corollary of the Parseval equation for w.a.p. functions:

THEOREM 1. $M_{x}\left[|\tilde{\mu}(x)|^{2}\right]=\sum_{y \in G^{*}}|\mu\{y\}|^{2}$.

Here the mean value $M(f)=M_{x} f(x)$ of a w.a.p. function $f$ may be defined as the (necessarily unique) constant that is the uniform limit of convex combinations of translates of $f$. In particular: $M$ is a linear functional. If $f$ is almost periodic, $M(|f|)=0$ only when $f=0$. $M_{x}(x, y)=0$ if $y \neq 0,=1$ if $y=0$. When $G$ is the additive group of the reals $\left(R_{1}\right), M$ has the representation

$$
M(f)=\lim L^{-1} \int_{0}^{L} f(x) d x .
$$

Proof of Theorem 1. Given a w.a.p. function $f(x)$ on $G$ with Fourier coefficients $a(y)=M_{x}[(x, y) f(x)]$, the Parseval equation reads:

Presented to the Society, December 28, 1953; received by the editors October 1 , 1953 and, in revised form, June 21, 1954.

1 This research was supported in part by the United States Air Force under Contract No. AF 18 (600)-679, monitored by the Office of Scientific Research.

${ }^{2}$ Numbers in brackets refer to the bibliography at the end of the paper. 


$$
M\left[|f|^{2}\right]=\sum_{\nu \in \sigma^{*}}|a(y)|^{2} .
$$

When $f(x)$ has the form $\tilde{\mu}(x)$ it remains to establish:

LEMma. $a(y)=\mu\{y\}$.

It is sufficient to show that $a(0)=M(\tilde{\mu})=\mu\{0\}$, for the general case follows on translation of $\mu$. When $G=R_{1}$, a standard Fubini and dominated convergence argument yields

$$
M_{x} \int_{-\infty}^{\infty}(x,-y) d \mu(y)=\int_{-\infty}^{\infty} M_{x}(x,-y) d \mu(y)=\mu\{0\} .
$$

The difficulty in general groups is that the mean value is represented as the limit of a directed set of convex combinations of translation operators rather than a sequence. However, it is clear that $M(\tilde{\mu})$ $=\mu\{0\}$ for a discrete measure $\mu(\nu=0)$, owing to uniform convergence of the series $\sum_{n}\left(x,-y_{n}\right) \mu\left\{y_{n}\right\}$. Hence we need only show that $M(\widetilde{\nu})=0$ for a continuous measure $\nu$.

Given any compact set $C^{*}$ in $G^{*}$ containing 0 as an interior point, write $\widetilde{\nu}(x)=f_{1}(x)+f_{2}(x)$, where

$$
f_{1}(x)=\int_{C^{*}}(x,-y) d \nu(y) ; \quad f_{2}(x)=\int_{G^{*}-C^{*}}(x,-y) d \nu(y) .
$$

Since $\left|M\left(f_{1}\right)\right| \leqq\left\|f_{1}\right\|_{\infty} \leqq|\nu|\left(C^{*}\right)$ is arbitrarily small for sufficiently small $C^{*}$, we need only show that $M\left(f_{2}\right)=0$. But it is well known (cf. $\left[5\right.$, p. 146]) that one can find a function $g$ in $L^{1}(G)$ such that $\tilde{g}(y)$ $=\int_{G}(x, y) g(x) d x$ vanishes on $G^{*}-C^{*}$ and $\tilde{g}(0)=1$. Consider the convolution $\left(f_{2} * g\right)(x)=\int_{G} f_{2}(x-t) g(t) d t=\int_{G^{*}-C^{*}}(x,-y) \tilde{g}(y) d \nu(y)=0$. Since $M\left(f_{2}\right)$ is a uniform limit of convex combinations of translations $f_{2}(x-t)$ of $f_{2}(t)$,

$$
0=\int_{G} M\left(f_{2}\right) g(t) d t=M\left(f_{2}\right) \tilde{g}(0)=M\left(f_{2}\right) .^{3} \text { q.e.d. }
$$

Theorem 1 for the real line is the intersection of several theorems of Wiener [7]. An immediate corollary is

Theorem 2 (Beurling-Helson). If $|\tilde{\mu}(x)| \equiv 1$, then

$$
\sum_{\nu \in G^{*}}|\mu\{y\}|^{2}=1
$$

In effect we have shown that 0 is not in the "point" spectrum of $f_{2}$, but an obvious modification of the argument shows that 0 lies in no part of the spectrum of $f_{2}$ (cf. [2]). 
Suppose that $\tilde{\mu}(x)$ is almost periodic. Then the difference $\tilde{\nu}(x)$ is an almost periodic function all of whose Fourier coefficients $a(y)$ vanish. Then $M\left(|\tilde{\nu}|^{2}\right)=0$, whence $\tilde{\nu}=0$, and then $\nu=0$ by the uniqueness theorem for Fourier-Stieltjes transforms. We thus obtain a result established by Segal [6] (and extended by Hewitt [4]) only for a special class of groups in which the mean value has a sequential limit representation:

Theorem 3. If $\tilde{\mu}(x)$ is almost periodic, $\mu$ is discrete $(\nu=0)$.

\section{BIBLIOGRAPHY}

1. W. F. Eberlein, Abstract ergodic theorems and weak almost periodic functions, Trans. Amer. Math. Soc. vol. 67 (1949) pp. 217-240.

2. - Spectral theory and harmonic analysis, Proceedings of the Symposium on Spectral Theory and Differential Problems, Stillwater, Oklahoma, 1951, pp. 209219.

3. H. Helson, Isomorphisms of Abelian group algebras, Arkiv för Matematik vol. 2 (1953) pp. 475-487.

4. E. Hewitt, Functions as Fourier-Stieltjes transforms, Proc. Amer. Math. Soc. vol. 4 (1953) pp. 663-670.

5. L. H. Loomis, $A$ n introduction to abstract harmonic analysis, New York, 1953.

6. I. E. Segal, The class of functions which are absolutely convergent Fourier transforms, Acta Sci. Math. Szeged. vol. 12 (1950) pp. 157-161.

7. N. Wiener, The Fourier integral, Cambridge, 1933.

UNIVERSITY OF WISCONSIN 